\title{
A Nafion Coated Capacitive Humidity Sensor on a Flexible PET Substrate
}

\author{
Christos Sapsanis, Ulrich Buttner, Hesham Omran, Youssef Belmabkhout, Osama Shekhah, Mohamed \\ Eddaoudi, Khaled Nabil Salama \\ King Abdullah University of Science and Technology (KAUST), Thuwal, 23955-6900, Saudi Arabia \\ khaled.salama@kaust.edu.sa
}

\begin{abstract}
This paper reports a simple and low-cost technique for fabricating low-power capacitive humidity sensors without the use of a cleanroom environment. A maskless laser engraving system was utilized to fabricate two different gold electrode structures, interdigitated electrodes and Hilbert's fifth-order fractal. The capacitive structures were implemented on a flexible PET substrate. The usage of Nafion, a well-known polymer for its hydrophilic properties as a sensing film, was attempted on the PET and outperformed the current efforts in flexible substrates. Its humidity sensing properties were evaluated in an automated gas setup with a relative humidity (RH \%) ranging from $15 \%$ to $95 \%$.
\end{abstract}

Keywords-Flexible humidity sensor, Nafion, PET, capacitive structures, laser engraving.

\section{INTRODUCTION}

Humidity sensing has attracted interest because of its many applications in environmental monitoring and industry. High humidity levels influence agriculture, for example plant diseases such as fungi and molds flourish, and long-term storage of grains can be affected negatively under humid conditions. Furthermore, moisture and condensation can adversely influence the quality of food products. Thus, high levels of humidity should be detected immediately [1] for cleaning and drying surfaces affected by moisture. Moreover, in industry humidity monitoring is required to ensure the continuity of end product quality and to minimize material waste. There are applications in medicine; where they are used, for instance, in respiratory equipment [2].

Different materials were attempted to be used as sensitive films in the literature. The materials employed can be polymers [3], carbon nanomaterials [4], metal organic frameworks [5], metal-oxide semiconductors [6]. Materials with positive and negative aspects relative to the sensor requirements can be selected based on what trade-offs the application can allow. Polymeric materials attract particular interest, as they can lower the sensor price more than other chemical substances. Additionally, they can operate at room temperature, which means less complicated instrumentation and no power consumption, as it is not necessary to use an element (e.g., a micro-heater) to increase the operating temperature [7].

Capacitive structures can be utilized as transducers and were selected due to their simple design. The preference for these kind of sensors is also related to the simple circuitry that can be used for capacitance readouts [8-10], thus providing an opportunity to produce a fully integrated microsystem.

The high cost of cleanroom processes and Si substrates have encouraged us to focus on reducing the cost and simplifying the fabrication of our sensor. PET is a plastic organic film used extensively for its flexibility and low cost. Previous efforts on flexible substrates were demonstrated [11-15] with different fabrications processes (Lithography, Inkjet-printing). This paper introduces laser engraving for fabricating transducers. We preferred sputtering Au on PET as in [16], instead of inkjet printing silver electrodes, because of its purity the precious metal achieves a lower dissipation factor than conductive ink. As a result, a more efficient capacitive structure can be produced in a trade-off of a more costly electrode's material. The same transducers were also used as cardio-vascular risk marker biosensor [17]. There are also some interesting efforts on flexible humidity sensors for RFID tags [18], ppm level moisture [19] and high humidity levels [20] detection.

The working principle of this sensor type is related to the relative permittivity of the sensitive film. The high dielectric constant of water (roughly 78 at room temperature) has an effect on the film, whose permittivity is significantly lower in most of the cases. Therefore, during the water vapor absorption or release under different humidity levels, there is an important variation in the film properties. This effect alternates the relative permittivity of the thin film, which eventually changes the output capacitance. Despite the fact that Nafion film is comprised mostly of hydrophobic $\left(-\mathrm{CF}_{2}-\mathrm{CF}_{2}-\right)$ groups, it exhibits hydrophilic behavior because ionic groups in the form of $\left(-\mathrm{SO}_{3}-\mathrm{H}+\right)$ allow conductive ions to migrate into the film upon moisture absorption [21]. Nation is a polyelectrolyte which allows hydrogen ions to migrate through the film [22].

\section{MATERIALS AND MethodS}

\section{A. Fabrication of capacitive structures}

The subtractive fabrication process illustrated in Figure 1 starts by sputtering $600 \mathrm{~nm}$ (Quorum 300T D) of gold on PET at a rate of $59 \mathrm{~nm} / \mathrm{min}$. The structures are then patterned with a mask-less laser etching allowing rapid prototyping. A 30W, 1.06-micron laser from Universal Laser Systems was used to pattern the structures. The parameters were experimentally optimized and results for higher power can be observed in Figure 2a, in which the Au was completely removed. Both IDEs and Hilbert's fractal structures were selected to have $100-\mu \mathrm{m}$ gap and finger widths and each sensor cell is $36-\mathrm{mm}^{2}$. Smaller resolution was not achieved for two reasons: Au peeled off frequently with the IDEs (Figure 2b), which caused discontinuities in the fingers and significantly decreased the 
capacitance; moreover, short circuit occurred in the Hilbert curve because of the complex structure (Figure 2c). The fabricated sensors and proof of their flexibility are depicted in Figure 3.

(a) Bare PET sheet
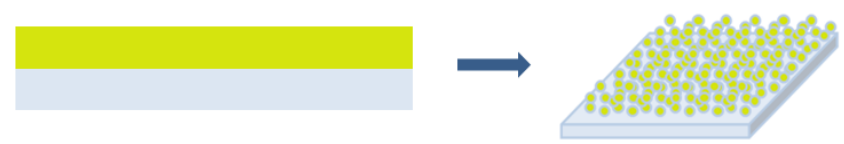

(b) Sputtering of $600 \mathrm{~nm} \mathrm{Au}$ (Rate: $59 \mathrm{~nm} / \mathrm{min}$ )

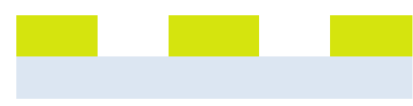

(c) Mask-less etching with laser engraving system

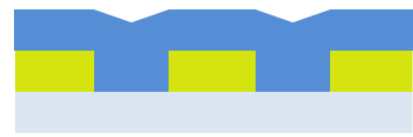

(d) Spin coating of sensing layer

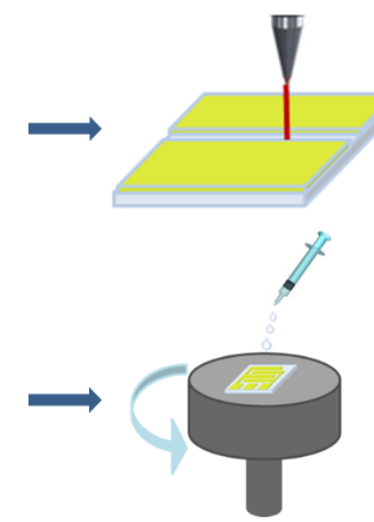

Figure 1: Fabrication steps.

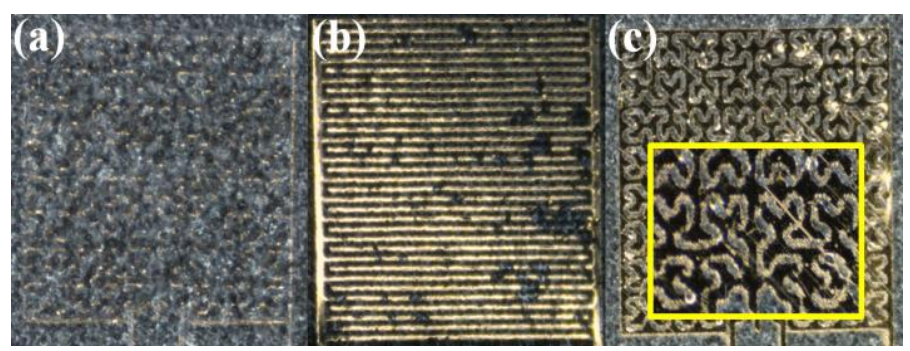

Figure 2: (a) Removal of $\mathrm{Au}$, (b) Au Peel off, (c) Short Circuit.

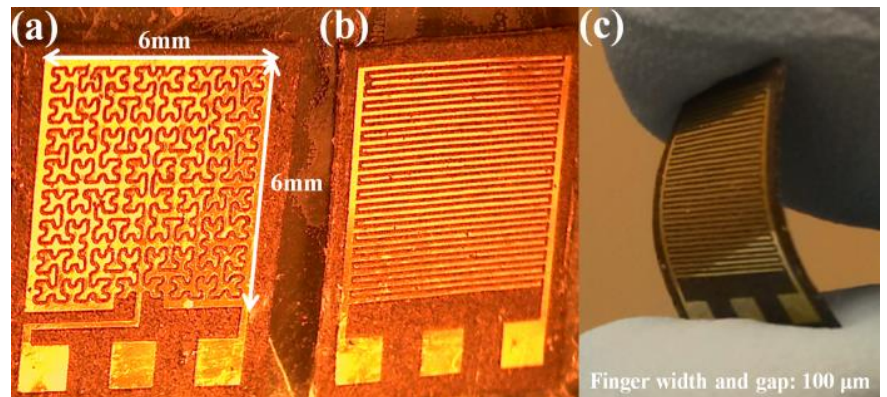

Figure 3: Fabricated capacitive structures (a) Hilbert fifth-order Fractal, (b) Interdigitated electrodes, (c) Proof of sensor flexibility.

As a last step in the humidity sensor fabrication, the capacitive structures were spin coated with Nafion at $500 \mathrm{rpm}$ for $5 \mathrm{sec}$ and $1000 \mathrm{rpm}$ for $30 \mathrm{sec}$. A $5 \mathrm{wt}$ \% Nation solution in a mixture of lower aliphatic alcohol and water from Sigma Aldrich was utilized.

\section{B. Measurement Setup}

In the humidity test setup it is important to show the stability of a sensor film. The experiments were conducted in an automated gas setup using LabVIEW [5]. Two paths were employed: dry $\mathrm{N}_{2}$ was used for diluting, and a carrier gas was used to generate water vapor through an immersed bubbler in a water chiller. The flow of the gas was controlled and measured by Mass Flow Controllers (MFCs). Stainless steel delivery lines were used on the setup with the exception of the vapor path, which was connected to a glass gas bubbler with special perfluoroalkoxy alkane (PFA) tubing for flexibility. The water vapor is collected in a stainless steel testing chamber. The testing cell encompasses two humidity sensors - a commercial sensor (Honeywell - HIH-4000 Series), and the fabricated sensor implemented one under test-with their values monitored by a Multimeter (Agilent 43301A) and an LCR meter (Agilent E4980A), respectively. A custom LabVIEW interface controls the MFCs and the whole experiment, as explained in Table 1. There is also a pressure check in the MFC of water vapor, which acts as a safety precaution shutting down the MFC in the case of exceeding the set limit. The whole system is depicted in Figure 4. The experiments were conducted at room temperature $\left(20^{\circ} \mathrm{C}\right)$, and the chiller was set in $17^{\circ} \mathrm{C}$ to produce the vapor.

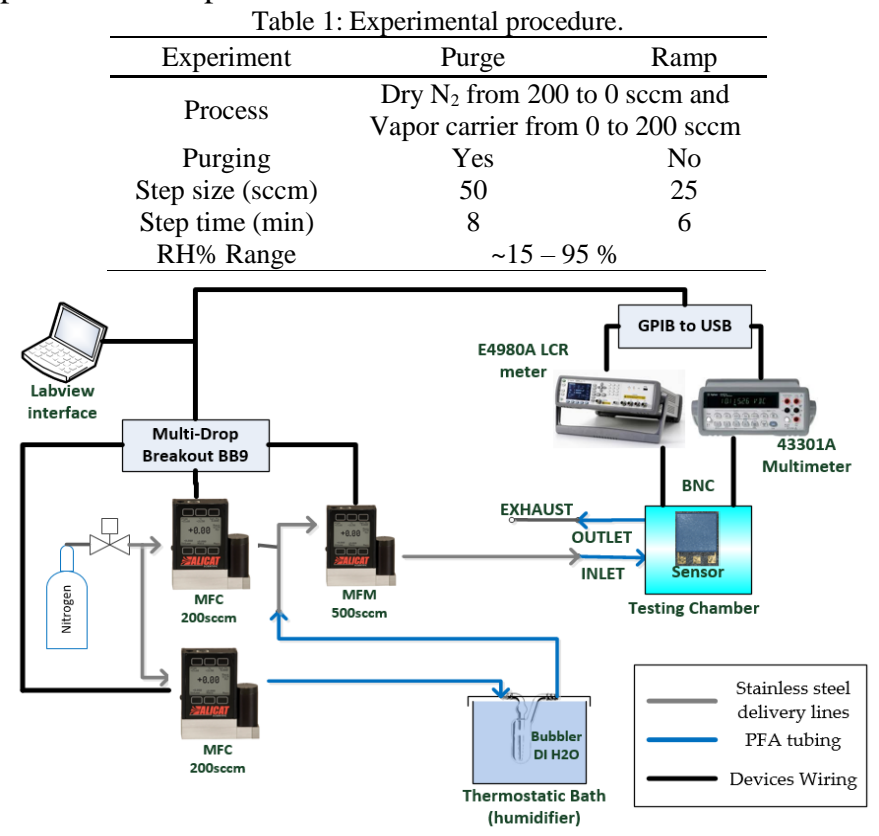

Figure 4: Schematic diagram of testing measuring setup.

\section{RESULTS AND DISCUSSION}

The first step after the fabrication of the capacitive structures was to evaluate the humidity sensing properties of the PET substrate. As it can be observed in Figure 5, the flexible polymer carrier achieves a sensitivity of 1.24 and $1.17 \mathrm{fF} / \mathrm{RH}$ for a $\mathrm{RH}<60 \%$ and 4.57 and $4.12 \mathrm{fF} / \mathrm{RH}$ for a $\mathrm{RH}>60 \%$ for the Hilbert's curve and IDEs structure respectively. Thus, the transducer has shown that even without any coating, it could have been used for humidity sensing. The low sensitivity is based on PET's nature, which is a well-known moisture barrier. Despite that fact, it can be still considered as a "sensitive" 
substrate especially for a $\mathrm{RH}>60 \%$. The fractal structure achieved a higher range compared to the IDEs, due to a higher initial capacitance value. Thus, Hilbert's curve was selected as capacitive structure for coating the polymer sensitive films.

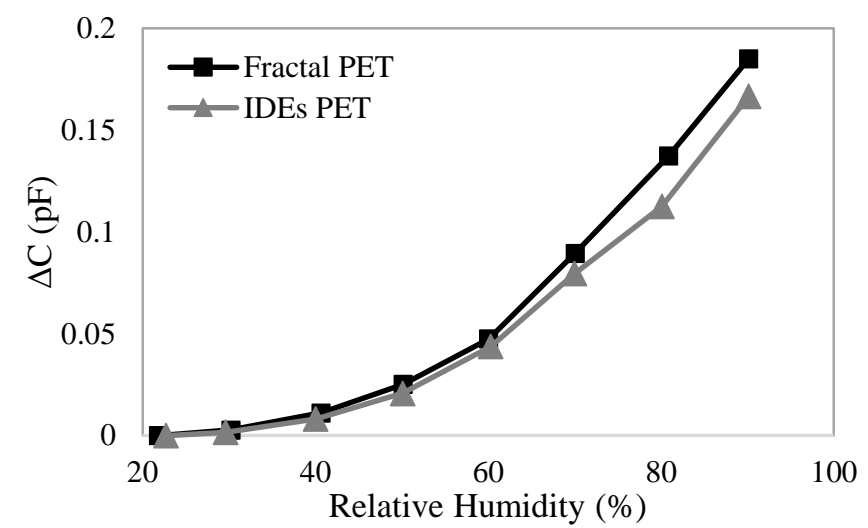

Figure 5: Humidity properties of PET substrate without any coated film. The capacitance was measured at $1 \mathrm{MHz}$.

Two types of experiments were conducted, ramp and purge, as shown in Figure 6 and described in Table 1. For the ramp experiment, the final value is marginally higher because it corresponds to a slightly higher humidity level than in the purge cycle. At higher humidity levels, even an $\mathrm{RH}$ increase of $1 \%$ can cause a significant increase in capacitance. Repeated experiments (three purge and one ramp) were conducted to prove that the results are reproducible.

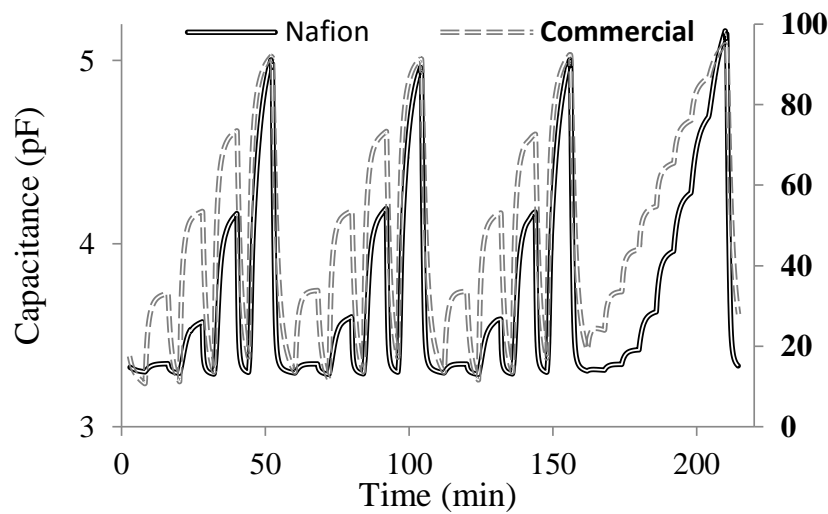

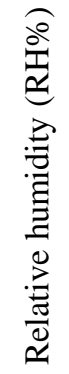

Figure 6: Three purge experiments followed by ramp experiment for Nafion film in fractal structure. Fabricated sensors' capacitance for Nafion was measured at $1 \mathrm{MHz}$ and uses the left $\mathrm{y}$-axis and relative humidity measurements for the commercial sensor uses the right $y$-axis.

Nafion exhibits a fast response and recovery time following almost simultaneously any changes of the commercial sensor, as depicted in Figure 6. The capacitance measurements are frequency dependent and the measurements were taken at four different frequencies $(1,10,100$ and $1000 \mathrm{kHz})$ at $1 \mathrm{~V}$. Capacitance measurements in low frequencies provide a long time for water dipoles to orient to a large extent in the material. Hence, the adsorbed water molecules in the sensitive film can be more polarized at lower frequencies. This fact can explain the humidity testing results in Figure 7 . Thus, the lower the frequency, the higher the sensitivity becomes and with a non- linear behavior especially over $60 \%$ of RH range.

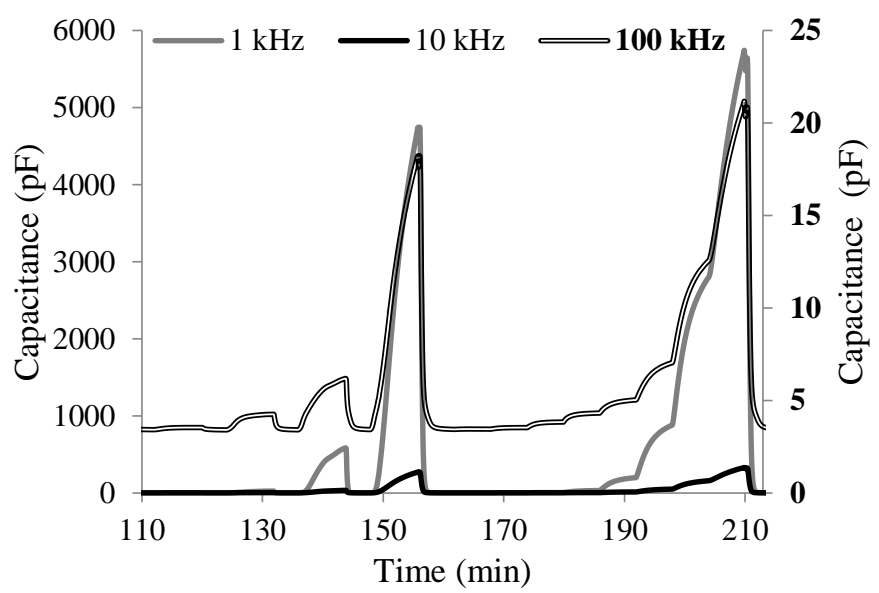

Figure 7: Nafion capacitance versus time at 1,10 and $100 \mathrm{kHz}$. The capacitance measured at $1 \mathrm{kHz}$ and $10 \mathrm{kHz}$ is associated with the left y-axis, while the 100 $\mathrm{kHz}$ uses the right $\mathrm{y}$-axis.

The results of the highest measured frequency $(1 \mathrm{MHz})$ were selected for extracting the sensitivity because they provide a more linear behavior, which is preferred in the characteristic curves of capacitance versus relative humidity. The humidity sensing behavior of Nafion is depicted in Figure 8 . The sensitivity was 12.27 and $40.86 \mathrm{fF} / \mathrm{RH}$ for $\mathrm{RH}<65 \%$ and $>65 \%$ respectively. Linear regression was conducted to calculate the characteristic curve, which is $\mathrm{y}=0.0005 \mathrm{x} 2-0.0263 \mathrm{x}+0.3738$ $\left(\mathrm{R}^{2}=0.998\right)$ and $\mathrm{y}=0.0399 \mathrm{x}-2.0116\left(\mathrm{R}^{2}=0.983\right)$ for $\mathrm{a}$ humidity range of $11 \%-65 \%$ and $65 \%-95 \%$ respectively.

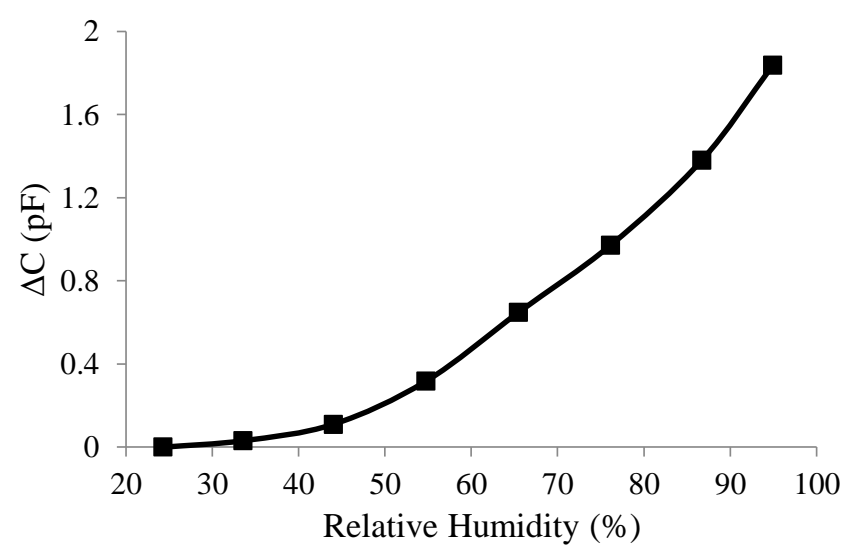

Figure 8: Capacitance difference measured at $1 \mathrm{MHz}$ versus relative humidity for Nafion sensor.

There are many efforts in the area of the flexible capacitive humidity sensors as described in Table 2, which shows a comparison of them with the current work. As for the fabrication methods, inkjet-printing is selected in [11-13], which despite the fact that shrinks the cost due to the low cost ink compared to the gold for electrodes material, it decreases the capacitors quality factor. Additionally, lithography was used in [12-13] and achieved higher resolution in the electrodes separation with a trade-off the important increase of the fabrication cost and process complexing by using mask for 
prototyping. In most of the cases, the measurements are in a specific frequency in comparison to our setup, which provides the sensitivity in range of frequencies from $1 \mathrm{kHz}$ to $1 \mathrm{MHz}$. Moreover, the testing setups used in the previous attempts achieved narrower humidity range.

As for the sensors sensitivity, the results of the current work outperform the previous efforts mentioned in [11-15]. This is primarily due to the fact that Nafion is well known for its hydrophilicity, which enhances the sensor's sensitivity due to the PET substrate. At higher levels of humidity (above $65 \%$ ), the water absorption increases importantly with a direct effect on the sensors sensitivity, which follows a different linear curve with a larger slope. This does not occur for the other efforts, where an almost linear behavior is observed for all the humidity range.

\section{CONCLUSION}

A promising method for fast and maskless fabrication of capacitive humidity sensors was described in this work. A subtractive method using laser engraving was introduced in order to avoid the expensive cleanroom usage. Moreover, PET was selected as a substrate coated with the hydrophilic polymer, Nafion. A wide range of RH\% measurements were extracted by the automated testing setup providing a clear evaluation of the sensors' humidity sensing capabilities. Nafion achieved a high sensitivity showing linear behavior above an $\mathrm{RH}$ of $65 \%$, and slightly non-linear behavior below it. The results outperformed the sensitivity of the aforementioned approaches in the flexible humidity sensors area.

\section{REFERENCES}

[1] Farahani, Hamid, Rahman Wagiran, and Mohd Nizar Hamidon. "Humidity sensors principle, mechanism, and fabrication technologies: A comprehensive review." Sensors 14.5 (2014): 7881-7939.

[2] Righettoni, Marco, Antonio Tricoli, and Sotiris E. Pratsinis. "Si: WO sensors for highly selective detection of acetone for easy diagnosis of diabetes by breath analysis." Analytical chemistry 82.9 (2010): 3581-3587.

[3] Sakai, Y., Y. Sadaoka, and M. Matsuguchi. "Humidity sensors based on polymer thin films." Sensors and Actuators B: Chemical 35.1 (1996): 85-90

[4] Zhang, Yongsheng, et al. "Quartz crystal microbalance coated with carbon nanotube films used as humidity sensor." Sensors and Actuators A: Physical 120.1 (2005): 142-146.

[5] Sapsanis, Christos, et al. "Insights on Capacitive Interdigitated Electrodes Coated with MOF Thin Films: Humidity and VOCs Sensing as a Case Study." Sensors 15.8 (2015): 18153-18166.
[6] Song, Seok-Ho, et al. "Metal-oxide-semiconductor field effect transistor humidity sensor using surface conductance." Applied Physics Letters 100.10 (2012): 101603.

[7] Claramunt, S., et al. "Flexible gas sensor array with an embedded heater based on metal decorated carbon nanofibres." Sensors and Actuators B: Chemical 187 (2013): 401-406.

[8] Omran, Hesham, Muhammad Arsalan, and Khaled N. Salama. "An integrated energy-efficient capacitive sensor digital interface circuit." Sensors and Actuators A: Physical 216 (2014): 43-51.

[9] Tan, Zhichao, et al. "A 1.2-V 8.3-nJ CMOS humidity sensor for RFID applications." Solid-State Circuits, IEEE Journal of 48.10 (2013): 2469 2477.

[10] Ha, Hyunsoo, et al. "12.6 A 160nW 63.9 fJ/conversion-step capacitanceto-digital converter for ultra-low-power wireless sensor nodes." SolidState Circuits Conference Digest of Technical Papers (ISSCC), 2014 IEEE International. IEEE, 2014

[11] Altenberend, Ulrike, et al. "Towards fully printed capacitive gas sensors on flexible PET substrates based on Ag interdigitated transducers with increased stability." Sensors and Actuators B: Chemical 187 (2013): 280 287.

[12] Lee, Chi-Yuan, Guan-Wei Wu, and Wei-jung Hsieh. "Fabrication of micro sensors on a flexible substrate." Sensors and Actuators A: Physical 147.1 (2008): 173-176.

[13] Mattana, Giorgio, et al. "Woven temperature and humidity sensors on flexible plastic substrates for e-textile applications." Sensors Journal, IEEE 13.10 (2013): 3901-3909.

[14] Mahboob, Md Rahat, et al. "A low cost polyimide based metal oxide film RH sensor." Industrial and Information Systems (ICIIS), 2014 9th International Conference on. IEEE, 2014.

[15] Molina-Lopez, Francisco, et al. "Fully inkjet-printed parallel-plate capacitive gas sensors on flexible substrate." Sensors, 2012 IEEE. 2012.

[16] Su, Pi-Guey, and Chao-Shen Wang. "Novel flexible resistive-type humidity sensor." Sensors and Actuators B: Chemical 123.2 (2007): 1071-1076.

[17] Sivashankar, S., et al. "Flexible low-cost cardiovascular risk marker biosensor for point-of-care applications." Electronics Letters 51.22 (2015): 1746-1748

[18] Feng, Yi, et al. "Low-cost printed chipless RFID humidity sensor tag for intelligent packaging." Sensors Journal, IEEE 15.6 (2015): 3201-3208.

[19] Islam, Tarikul, Md Rahat Mahboob, and Shakeb A. Khan. "A Simple MOX Vapor Sensor on Polyimide Substrate for Measuring Humidity in ppm Level." Sensors Journal, IEEE 15.5 (2015): 3004-3013.

[20] Islam, Tarikul, Zia Ur Rahman, and Subhas Chandra Mukhopadhyay. "A novel sol-gel thin-film constant phase sensor for high humidity measurement in the Range of 50\%-100\% RH." Sensors Journal, IEEE 15.4 (2015): 2370-2376.

[21] Tailoka, F., D. J. Fray, and R. V. Kumar. "Humidity sensing under aggressive conditions using nafion conductors." Ionics 6.5-6 (2000): 383388.

[22] Feng, Chang-Dong, et al. "Humidity sensing properties of Nation and solgel derived $\mathrm{SiO}$ 2/Nafion composite thin films." Sensors and Actuators B: Chemical 40.2 (1997): 217-222.

Table 2. Comparative table of humidity sensitivity for flexible substrates (Low RH $<65 \%$, High RH $>65 \%$ ).

\begin{tabular}{|c|c|c|c|c|c|c|c|c|c|c|c|c|c|c|}
\hline \multirow{3}{*}{ Film } & \multirow{3}{*}{$\begin{array}{l}\text { Electrodes } \\
\text { Type }\end{array}$} & \multirow{3}{*}{$\begin{array}{l}\text { Substrate } \\
\text { Material }\end{array}$} & \multirow{3}{*}{$\begin{array}{l}\text { Electrode } \\
\text { Material }\end{array}$} & \multirow{3}{*}{$\begin{array}{c}\text { Fabrication } \\
\text { Method }\end{array}$} & \multirow{3}{*}{$\begin{array}{l}\text { Humidity } \\
\text { Range }\end{array}$} & \multicolumn{8}{|c|}{ Sensitivity (fF/RH\%) } & \multirow{3}{*}{ Ref } \\
\hline & & & & & & \multicolumn{2}{|c|}{$1 \mathrm{kHz}$} & \multicolumn{2}{|c|}{$10 \mathrm{kHz}$} & \multicolumn{2}{|c|}{$100 \mathrm{kHz}$} & \multicolumn{2}{|c|}{$1 \mathrm{MHz}$} & \\
\hline & & & & & & $\mathrm{L}$ & $\mathrm{H}$ & $\mathrm{L}$ & $\mathrm{H}$ & $\mathrm{L}$ & $\mathrm{H}$ & $\mathrm{L}$ & $\mathrm{H}$ & \\
\hline Nafion & $\begin{array}{l}\text { Fractals } \\
\text { (100 um) }\end{array}$ & PET & $\mathrm{Au}$ & $\begin{array}{c}\text { Laser } \\
\text { Engraving }\end{array}$ & $20 \%-95 \%$ & 3634 & 188160 & 210 & 10727 & 29.72 & 547.86 & 12.27 & 40.86 & $\begin{array}{r}\text { This } \\
\text { work }\end{array}$ \\
\hline PET sub. & IDEs (100um) & PET & $\mathrm{Ag}$ & Inkjet-printing & $0 \%-70 \%$ & \multicolumn{2}{|c|}{2.92} & & - & \multicolumn{2}{|c|}{2.03} & \multicolumn{2}{|c|}{-} & {$[11]$} \\
\hline Polyimide & IDEs (20 um) & Parylene & $\mathrm{Au}$ & Lithography & $25 \%-95 \%$ & \multicolumn{6}{|c|}{30 (frequency not mentioned) } & & & [12] \\
\hline Kapton sub. & IDEs (10 um) & Kapton & $\mathrm{Au}$ & Lithography & $30 \%-78 \%$ & - & - & & - & \multirow{2}{*}{\multicolumn{2}{|c|}{$\begin{array}{r}7.292 \\
0.593 \\
\end{array}$}} & \multicolumn{2}{|c|}{-} & \multirow{2}{*}[13]{} \\
\hline Kapton sub. & IDEs (80 um) & Kapton & $\mathrm{Ag}$ & Inkjet-printing & $25 \%-84 \%$ & - & - & & - & & & - & - & \\
\hline $\begin{array}{l}\gamma-\mathrm{Al}_{2} \mathrm{O}_{3} \\
\& \text { PI sub. } \\
\end{array}$ & Parallel plate & $\begin{array}{l}\text { UPILEX-S } \\
\text { polyimide } \\
\end{array}$ & $\mathrm{Ag}$ & Sol-gel & $5 \%-85 \%$ & \multicolumn{2}{|c|}{4044} & & - & \multicolumn{2}{|r|}{-} & \multicolumn{2}{|c|}{-} & [14] \\
\hline $\mathrm{CAB}$ & Parallel plate & PET & $\mathrm{Ag}$ & Inkjet-printing & $15 \%-72 \%$ & \multicolumn{2}{|r|}{-} & & - & \multicolumn{2}{|c|}{9.3} & \multicolumn{2}{|c|}{-} & [15] \\
\hline
\end{tabular}

